\title{
Disrupted salience network dynamics in Parkinson's disease patients with impulse control
}

\section{disorders}

Irene Navalpotro-Gomez ${ }^{\mathrm{a}, 1}$, Jinhee Kim ${ }^{\mathrm{b}}$, Pedro M. Paz-Alonso ${ }^{\mathrm{c}}$, Delgado-Alvarado $\mathrm{M}^{\mathrm{a}, \mathrm{d}, \mathrm{e}, \mathrm{f}}$, Quiroga-Varela $\mathrm{A}^{\mathrm{a}, \mathrm{g}}$, Jimenez-Urbieta $\mathrm{H}^{\mathrm{a}}$, Carreiras $\mathrm{M}^{\mathrm{c}, \mathrm{h}}$, Antonio P Strafella ${ }^{\mathrm{b}, \mathrm{i}, \mathrm{j}}$, Maria Cruz Rodriguez-Oroz ${ }^{\mathrm{a}, \mathrm{c}, \mathrm{g}, \mathrm{k}, *}$

${ }^{a}$ Neurodegenerative Disorders Area, Biodonostia Health Research Institute, San Sebastián, Spain

${ }^{\mathrm{b}}$ Research Imaging Centre, Campbell Family Mental Health Research Institute, Centre for Addiction and Mental Health, University of Toronto, Toronto, Ontario, Canada

${ }^{\mathrm{c}}$ Basque Center on Cognition, Brain and Language (BCBL), San Sebastián, Spain

${ }^{\mathrm{d}}$ Neurology Department, Sierrallana Hospital, Torrelavega, Spain

${ }^{\mathrm{e}}$ IDIVAL, Valdecilla Biomedical Research Institute, Santander, Spain

${ }^{\mathrm{f}}$ Biomedical Research Networking Center for Mental Health (CIBERSAM), Madrid Spain

${ }^{\mathrm{g}}$ Neuroscience Area, CIMA, University of Navarra, Navarra, Spain

${ }^{\mathrm{h}}$ Ikerbasque (Basque Foundation for Science), Bilbao, Spain

${ }^{\mathrm{i}}$ Brain, Imaging and Behaviour- Systems Neuroscience Division, Krembil Research Institute, UHN, University of Toronto, Ontario, Canada

${ }^{\mathrm{j}}$ Morton and Gloria Shulman Movement Disorder Unit \& E.J. Safra Parkinson Disease Program, Neurology Division, Department of Medicine, Toronto Western Hospital, UHN, University of Toronto, Ontario, Canada

${ }^{\mathrm{k}}$ Department of Neurology, Clinica Universidad de Navarra, Universidad de Navarra, Pamplona, Spain

*Corresponding author: Maria C Rodriguez-Oroz, Department of Neurology, Clinica Universidad de Navarra, University of Navarra, Pamplona, Spain. Tel.: +34948255400 Ext 4521.

${ }^{1}$ Present address: Servei de Neurologia, Hospital del Mar, Parc de Salut Mar-IMIM, Barcelona, Spain 
E-mail addresses: INavalpotro@parcdesalutmar.cat (I. Navalpotro-Gomez), jinhee.kim@camhpet.ca (J. Kim), p.pazalonso@bcbl.eu (P.M. Paz-Alonso), manueldelgadoalv@gmail.com (M. Delgado-Alvarado), aquiroga@unav.es (A. Quiroga-Varela), haritzjimenez@hotmail.com (H. Jimenez-Urbieta), m.carreiras@bcbl.eu (M. Carreiras), antonio.strafella@uhn.ca (A. Strafella), mcroroz@unav.es (M.C. Rodriguez-Oroz).

\section{KEYWORDS}

Parkinson's disease, impulse control disorders, dynamic functional connectivity, graph theory, salience network. 


\section{ABSTRACT}

- Background: Dynamic functional network analysis may add relevant information about the temporal nature of the neurocognitive alterations in PD patients with impulse control disorders (PD-ICD). Our aim was to investigate changes in dynamic functional network connectivity (dFNC) in PD-ICD patients, and topological properties of such networks.

- Methods: Resting state fMRI was performed on 16 PD PD-ICD patients, 20 PD patients without ICD and 17 healthy controls, whose demographic, clinical and behavioral scores were assessed. We conducted a group spatial independent component analysis, sliding window and graph-theory analyses.

- Results: PD-ICD patients, in contrast to PD-noICD and HC subjects, were engaged across time in a brain configuration pattern characterized by a lack of between-network connections at the expense of strong within-network connections (State III) in temporal, frontoinsular and cingulate cortices, all key nodes of the salience network. Moreover, this increased maintenance of State III in PD-ICD patients was positively correlated with the severity of impulsivity and novelty seeking as measured by specific scales. While in State III, these patients also exhibited increased local efficiency in all the aforementioned areas.

- Conclusions: Our findings show for the first time that PD-ICD patients have a dynamic functional engagement of local connectivity involving the limbic circuit, leading to the inefficient modulation in emotional processing and reward-related decision-making. These results provide new insights into the pathophysiology of ICD in PD patients and indicate that the $\mathrm{dFC}$ study of fMRI could be a useful biomarker to identify patients at risk to develop ICD. 


\section{INTRODUCTION}

Impulse control disorders (ICDs), occur in at least 13.6\% of patients with Parkinson's disease (PD) on dopaminergic medication. They have been conceptualized as 'behavioral addictions' and are the result of abnormal modulation of the mesocorticolimbic dopamine system [1].

Dopaminergic depletion in PD mainly involves the nigrostriatal 'motor' loop, but also the ventral tegmental area (VTA) projecting to the ventral striatum (VS), amygdala and hippocampus (mesolimbic dopaminergic pathway), and to the orbitofrontal (OFC), frontoinsular, cingulate and prefrontal (PFC) cortices (mesocortical dopaminergic pathway). These regions are critically involved in the modulation of emotional processing, hedonic evaluation and rewardrelated decision-making [2], all closely associated with ICDs. However, the pathophysiology of ICDs in PD patients is not well understood. Evidence from metabolic [3], functional magnetic resonance imaging (fMRI) $[4,5]$ and morphometric neuroimaging studies [6] has demonstrated abnormalities within the mesocorticolimbic circuit in patients with PD and ICD (PD-ICD) although not always in the same direction and regions [7]. In particular, resting state fMRI (rsfMRI) studies, have produced controversial results in PD-ICD patients, with both weaker [4] and stronger [8] functional connectivity (FC) detected in cortical areas of the limbic circuit, as well as disrupted functional coupling in brain-wide networks such as the salience, executive and default-mode networks $[9,10]$. In addition, there have been very few studies into the topological characteristics of brain networks in these patients using graph theory analysis [11,12].

Significantly, most rs-fMRI studies on PD patients have investigated FC patterns as a static phenomenon, while recent evidence shows that FC may vary over time [13]. Such temporal fluctuations can be captured by dynamic functional network connectivity (dFNC) studies [14], providing greater insight into the fundamental properties of brain networks. In PD patients, 
temporal fluctuations in FC have been described as closely related to the severity of motor [15] and cognitive impairment $[16,17]$, but its relation with impulsivity has not been studied. Given that previous static FC studies have shown that dopaminergic firing is able to alter limbic cortical signals to the VS, impairing the ability to change behavioral focus in response to change in stimulus salience $[8,9,18]$, a dFNC approach may add relevant information as it represents the temporal changes of these connections [13]. For this reason, we hypothesized that PD-ICD patients would show specific dynamic functional connectivity (dFNC) changes in particular areas of the salience network/reward processing, possibly because these patients may continue to focus on those reward stimuli that have primarily induced the dopamine release, looping the cerebral areas implicated in hedonic evaluation of the stimulus and reward-based decision making. Therefore, the aim of this study was to evaluate differences in dFNC and topological metrics in PD patients with and without ICDs, utilizing a combination of dFNC analyses and graph-theory approaches (GTA) during rs-fMRI.

\section{MATERIALS AND METHODS}

\subsection{Subjects}

The final study sample consisted of 62 participants took part from three groups: 20 PD-ICD patients, 22 PD patients without ICD symptoms (PD-noICD) and 20 healthy controls (HC). Participants in each group were matched on age, sex, education and premorbid Intelligence Quotient. All PD patients were diagnosed according to the UK Parkinson's Disease Society Brain Bank criteria and recruited from the Movement Disorders Unit at the Hospital Donostia. A neurologist and a psychiatrist detected and confirmed ICD diagnosis based on the DSM-V and on the Questionnaire for Impulsive-Compulsive Disorders in Parkinson's Disease (QUIP) [19]. ICD severity was scored using the QUIP-Rating Scale (QUIP-RS) [20]. The score of each ICD 
subtype in every patient was above the established cut-off value (Supplementary Table 1). We excluded patients with dementia, cognitive impairment following the level II guidelines of the Movement Disorders Society (MDS) Criteria Task Force [21], dyskinesias, previous brain surgery, and patients with past ICD no longer symptomatic. HCs were recruited from the Basque Center on Cognition, Brain and Language (BCBL) participant pool. They were also evaluated by a neurologist and a psychiatrist and subjects with any neurological or psychiatric disorder were excluded. In addition, based on general population impulsivity scales, HCs with high scores at the higher end of the impulsivity scale were also excluded. Participants were evaluated using a battery of tests for motor, behavior and cognition (Table 1 and Supplementary Table 2). PD patients were studied under the effect of their usual dopaminergic medication. The study was approved by the Local Ethics Committee and written informed consent was obtained from all subjects prior to their enrollment.

\subsection{MRI data acquisition and preprocessing}

The rs-fMRI and structural data were acquired on a 3T Siemens Magnetom TIM Trio MRI scanner (Siemens Medical Solutions, Erlangen, Germany) using a 32-channel head coil. Further information on the MRI data acquisition and preprocessing is included in the Supplementary Materials. Briefly, the Conn Functional Connectivity Toolbox v.17.f [22] running on Matlab ${ }^{\mathrm{TM}}$ 9.1 (release R2016b) was used to conduct standard preprocessing routines and analyses. None of the participants included in the study sample had white matter abnormalities in their T2weighted MRI images.

\subsection{Motion correction}

To minimize the potential effects of head movements on dFNC results, we applied stringent inclusion criteria [14] following previous articles published on $\mathrm{dFCN}$ in PD populations [15,16] 
(see details in Supplementary Text). Briefly, subjects with a mean framewise displacement (FD)

$>0.5 \mathrm{~mm}$ or with a maximum displacement $>3 \mathrm{~mm}$ in translation indexes $\mathrm{x}, \mathrm{y}$, or $\mathrm{z}$ or $>3^{\circ}$ in rotation indexes were excluded from the analysis. Thus, nine participants (4 PD-ICDs, 2 PDnoICDs, $3 \mathrm{HCs}$ ) were excluded. In addition, there were no statistical root mean square (RMS) movement differences between PD-ICD (0.1 \pm 0.09$)$, PD-noICD $(0.08 \pm 0.09)$ and HC $(0.12 \pm 0.1)$ groups $(\mathrm{p}=0.47)$.

\subsection{Identification of intrinsic connectivity networks}

The data were decomposed into functional networks by applying a spatial group independent component analysis (ICA) using the Group ICA of the fMRI Toolbox (GIFT v4.0a; http://icatb.sourceforge.net We chose relatively high model order ICA, each individual's functional data was reduced to a total of 134 independent components (ICs) through a principal component reduction and subsequently, the concatenated subject-reduced data was reduced to 100 ICs at the group level using the expectation maximization algorithm [23] included in GIFT.

Of the $100 \mathrm{ICs}, 40$ were identified as meaningful by two independent reviewers (I.N. and J.K.), based on expectations that resting state networks should exhibit peak activations in gray matter, low spatial overlap with known vascular, ventricular, motion, and susceptibility artifacts, and time-courses dominated by low frequency fluctuations and with a high dynamic range [24]. Also, to examine potential between-group differences in gray matter we used participants' T1weighted images to run Freesurfer's mri_glmfit [25] on gray-matter volume, cortical thickness, and surface area. We found no significant differences in any of the group comparisons (PD-ICDs vs. PD-noICDs, PD-ICDs vs. HCs, PD-noICD vs. HCs).

Using spatial correlation values between ICs and the template [26], we classified the selected components into the following seven functional networks: basal ganglia [BG], auditory [AUD], 
sensorimotor [SMN], visual [VIS], cognitive-executive [ECN, including language and salience networks], default mode [DMN] and cerebellar networks (Figure 1A). The cognitive control network included IC components involved in directing and monitoring behavior and in mediating memory and language functions because of their overlap in several areas, as in previous works in the field [27].Pairwise Pearson's correlations were computed and converted to z-scores using Fisher's z-transformation to create the statistical FNC matrix (Figure 1B and 1C). For further details in identification of intrinsic connectivity networks see Supplementary Text.

\subsection{Dynamic functional network connectivity (dFNC) analysis}

dFNC was examined using the GIFT toolbox for dynamic FNC applying two approaches: a sliding window approach (changes in FNC across time) and k-means clustering (extracting reoccurring FNC patterns) First, we used a sliding-window approach, in which a sliding time window of the 22-repetition time (TRs) method was applied to each subject, with a Gaussian window alpha value of 3 and a step between windows of 1 TR, resulting in 270 consecutive windows. To promote sparsity in the estimations, a penalty was imposed on the L1 norm of the precision matrix. We also applied a k-means clustering algorithm to the $270 \mathrm{FC}$ window $\mathrm{FNC}$ matrices for all the subjects, which was iterated 150 times. To reduce redundancy between windows and computational demands, windows consisting of local maxima in functional connectivity variance were used. In addition, we used the L1 distance for k-means computing to combine similar FC matrices from the different windows. We used age, sex and depression scores as covariates, the latter due to the significant differences between the groups (Table 1). The covariates were included in the clustering algorithm using the GIFT toolbox (http://icatb.sourceforge.net/groupica.htm). Based on the elbow criterion of the cluster validity index, the optimal number of clusters was determined to be four $(k=4)$, the so-called states I, II, 
III and IV. Using the resultant four cluster centroids, all FNC matrices of each subject were then categorized as belonging to one of the four clusters or states based on their similarity to the cluster centroids. From these data, we obtained a state transition vector and final cluster centroids were obtained as the median of all state-assigned FCN matrices across time. The subject-specific centroid of each state was computed by calculating the median value of each FCN matrix for that state.

We used three different indices to examine the temporal properties of the dFNC states: (1) Fractional window (FW), the proportion of time spent in each state; (2) mean dwell time (DT), represents how long the participant remained in a certain state; (3) the number of transitions, the total number of changes between states. The differences between groups were assessed using an ANOVA, applying a LSD post-hoc test. Moreover, the FC differences between the groups in each FC state were investigated with the network-based statistic (NBS) tool. A p value FDR corrected for multiple comparisons was calculated. The significance of FC differences were tested using an ANOVA test for the three group differences and post-hoc pairwise comparisons. Overall, the $\mathrm{p}$ values were false discovery rate (FDR) corrected for multiple comparisons (i.e.: $\mathrm{q}$ values). See Supplementary Text for more details about dFNC analysis.

\subsection{Graph-theory parameter analysis}

Graph characteristics of the networks were analyzed using GRETNA software v 2.0.0 (http://www.nitrc.org/projects/gretna) in the FNC configuration. We investigated the topological characterization of brain connectivity, studying local (clustering coefficient, betweenness centrality and the local efficiency) and global (global efficiency and assortativity) metrics. For further details see Supplementary Text.

\subsection{Statistical analyses}


Statistical methods for clinical variables are detailed in Supplementary Text. The threshold for statistical significance was $\mathrm{p}<0.05$. We used a Pearson's correlation analysis to explore the associations between the temporal properties and the clinical measures in PD-ICD patients, applying a post-hoc Bonferroni correction for multiple comparisons (IBM SPSS Statistics v16.0).

\section{RESULTS}

\subsection{Sociodemographic and clinical data}

This analysis was carried out in 16 PD-ICD, 20 PD-noICD patients and $17 \mathrm{HC}$ (nine participants were excluded from the initial sample because of motion criteria). PD-ICD patients had significantly higher novelty seeking personality traits, impulsivity and depression scores than the other groups. There were no other clinical or cognitive differences between the groups (Table 1 and Supplementary Table 2).

\subsection{Intrinsic connectivity networks}

The spatial maps of the 40 ICs defined were established using the group ICA (see Figure 1 and Supplementary Table 3). Based on their anatomical and presumed functional properties, the ICs were grouped into the following networks: BG (IC 16, 22, 37); AUD (IC 34); SMN (IC 19, 4, 3, 54, 68, 24, 12); VIS (IC 25, 42, 90, 93, 59, 40, 26, 44, 45); ECN (IC 78, 49, 62, 92, 47, 18, 35), encompassing the language (IC 43, 79) and salience networks (IC 85, 39, 56); DMN (IC 15, 23, 88, 48, 66, 76); and the cerebellar network (IC 29, 31).

\subsection{Differences in dynamic FC}


A k-means clustering algorithm identified four different dFNC matrix centroid configurations or states that were recurrent throughout the rs-fMRI acquisition and across subjects (Figure 2A and 2B). The most frequent state (State III, $45 \%$ of acquisition time) appeared as a within-network FC configuration, particularly associated with the SMN, VIS and ECN networks which almost completely lacks between-network connectivity. By contrast, the other three states were more infrequent (State I 16\%; State II 19\%; State IV 19\%), and they displayed between-network FC configurations that included positive correlations between the SMN and VIS networks, as well as anti-correlations between the SMN and DMN, and between the ECN and DMN networks. When the $5 \%$ of the FC network with the strongest connections was represented, State III had the most positive within-network coupling (Figure 2C). In States I, II and IV we observed that $60 \%$ of connections were between networks (VIS-SM, SM-cerebellum, VIS-DMN, DMN-SM, ECNVIS, BG-VIS, BG-ECN, etc.). However, in State III we observed 38 stronger within- and between-network connections of which only $30 \%$ were between-network connections. Thus, State III was predominantly characterized by within-network connections (particularly connections between independent components located in the ECN, VIS and SM networks).

In terms of temporal properties, State III showed a trend to be more frequent (higher FW) in PDICD patients $(57 \pm 0.3)$ than in the PD-noICD subjects $(40 \pm 0.3)$ or the HCs $(39 \pm 0.2)(\mathrm{F}(2,50)=$ 2.41; $\mathrm{p}=0.07)$. In parallel, these patients showed a significantly larger mean DT in State III $(41.5 \pm 20.7)(F(2,50)=3.88 ; p=0.027)$, characterized by stronger local connectivity than PDnoICD subjects $(24.8 \pm 18.9 ; \mathrm{p}=0.028)$ and $\mathrm{HCs}(21.6 \pm 10.5 ; \mathrm{p}=0.013$ : Figure 3.1.; Supplementary Figure 1). There were no differences in transition number. When the FC differences were calculated between the PD-ICD, PD-noICD and HC groups for each dFNC state $(q<0.05$ FDR corrected: Supplementary Figure 2), we found that the within-network FC of the 
salience network was enhanced in PD-ICD patients in State III, specifically in the superior temporal lobe, frontoinsular and cingulate cortices ( $q<0.05$ FDR corrected).

\subsection{Relationship with clinical properties}

In PD-ICD patients, only in State III, the mean DT was positively correlated with the TCI-R NS4 sub-score (disorderliness: $\mathrm{r}(14)=0.519 ; \mathrm{p}=0.039)$ and with the non-planning sub-score of the BIS scale $(\mathrm{r}(14)=0.541 ; \mathrm{p}=0.030)$, and the mean percentage FW was correlated with the nonplanning sub-score of the BIS scale $(\mathrm{r}(14)=0.536 ; \mathrm{p}=0.032)$ and with the total score of the BIS scale $(\mathrm{r}(14)=0.561 ; \mathrm{p}=0.024$ : Figure 3.2$)$. These effects were not observed either in PD-noICD patients, or in HCs (ps $\geq 0.25$ ). In addition, dopaminergic medication (LEDD TOTAL $_{\text {and }}$ EED $_{\mathrm{DA}}$ ) was not correlated with any temporal property of State III ( $\mathrm{ps} \geq 0.34$ ).

\subsection{Graph topological properties}

We found no differences in global network metrics, yet when regional properties were considered. The PD-ICD patients had greater local efficiency than PD-noICD subjects (the average shortest path connecting all neighbors of a node) in the superior temporal lobe (BA 48; salience network, SAL; $\mathrm{p}=0.0440)$, frontoinsular $(\mathrm{BA} 6$; SAL; $\mathrm{p}=0.0384)$ and cingulate cortices (BA32; SAL; $\mathrm{p}=0.0160$ ), all of which belong to the salience network.

\section{DISCUSSION}

This is the first study investigating dFNC and graph properties in PD-ICD patients during rsfMRI. Our results reveal that in contrast to PD-noICD and HC subjects, PD-ICD patients were engaged across time in a brain configuration pattern characterized by a lack of between-network connections at the expense of strong within-network connections (State III) in temporal, frontoinsular and cingulate cortices, all key nodes in the salience network. Moreover, this 
increased maintenance of State III in PD-ICD patients, reflected by its temporal dFNC properties (FW and DT), was positively correlated with impulsivity (BIS scale) and novelty-seeking personality (TCI-R NS) ratings. Importantly, PD-ICD patients also exhibited increased local efficiency in all the aforementioned areas, suggesting brain reorganization in regions responsible for emotional processing and hedonic evaluation and associated with reward-related decision making.

FCs can be classified into long-range connections between different networks (between-network connectivity), allowing integration of long-distance information, and local connections within clusters of neurons (within-network connectivity) favoring local information processing. In our cohort, the dFNC analyses suggested four different connectivity patterns or states. State III was characterized by reduced expression of between-network coupling and a prominent withinnetwork connectivity, mainly in the SMN, VIS and ECN networks. Accordingly, State III could be interpreted as involving a lower degree of connectedness between regions whereas the other (States I, II and IV) showed stronger between-networks couplings.

Few studies have investigated dFNC in PD patients showing higher between-network FNC in PD patients than in HCs, interpreted as a potential compensatory mechanism [15] and reduced links between networks connectivity in PD patients with mild cognitive impairment [16,17], In our study, PD-ICD patients showed enhanced maintenance (DT) and a tendency towards remaining for higher proportion of time with functional connections (FW) in State III with respect to the other two groups. This confirms our hypothesis that altered temporal properties in dynamic connectivity is associated with ICD in PD patients. Thus, PD-ICD patients gravitate toward configurations with predominantly within-network FNC patterns, characterized by stronger connectivity between critical hubs that make up the salience network responsible for anchoring 
awareness through integration of contextual, hedonic, homoeostatic and interoceptive processes, and which partially overlaps with regions of the mesocorticolimbic circuit: the superior temporal lobe, and frontoinsular and cingulate cortices. The temporal predominance of this modular brain organization could reflect dopaminergic dysregulation of the emotional processing circuit in PDICD patients, in line with a proposed theory linking the relevance of a specific cognitive process to the length of time spent in the state that facilitates this cognitive aspect [28]. This may leave PD-ICD patients permanently focused on rewarded stimuli, an effect primarily due to dopamine overstimulation which leads to abnormal reward weighting. Importantly, our result is especially relevant considering that PD patients with and without ICD had no cognitive differences in any cognitive domain.

In addition, the FW and the DT in state III in PD-ICD patients were positively associated with the BIS scale, which measures different aspects of impulsivity, but also with the Novelty Seeking personality test evaluating exploratory activity in response to novel stimuli, highlighting the specificity of the findings. Accordingly, a recent static FC study demonstrated the presence of a specific pattern of intrinsic connectivity within and between the SAL, ECN and DMN at baseline in drug naïve PD patients who went on to develop ICD after treatment [10]. Our results emphasize the importance of dFNC studies, not only to analyze the pathophysiology of impulsivity in PD-ICD patients but also to assess its relevance in enhancing the drive to seek and explore novel stimuli. Such an effect deserves further study in drug-naïve PD patients, as it is a personality trait that could play a role in the development of ICDs.

Specifically, PD-ICD patients only appear to exhibit stronger local connections and increased local efficiency in areas that belong to the salience network. This is a large-scale limbicparalimbic network that encompasses cortical areas which play an important role in orienting 
attention to integrate salient, hedonic and interoceptive stimuli which facilitate goal-directed behavior, [29] such as the anterior cingulate cortex, bilateral insula, frontoinsular cortex, amygdala, VS, and substantia nigra/VTA [29]. Abnormal connectivity within this network has been associated with substance-related disorders and addictive behaviors, and interestingly, a recent $\mathrm{dFNC}$ study on the general population suggested that susceptibility to tempting stimuli is governed by individual differences in salience network dynamics [30].

Graph theoretical methods provide a mathematical framework to study the topological properties of networks, indicating that while global network measures may exhibit greater temporal stationarity, regional network measures may be more susceptible to local cell dynamics [31]. To date, only two studies have reported disruptions in regional topology in PD-ICD patients: one revealed altered properties of connections to the mesocorticolimbic system [12], while the other, demonstrated stable topological properties irrespective of the state of medication [11], which is relevant to our study. dFNC alterations in PD-ICD patients were accompanied by greater local efficiency in the PD-ICD group in areas of the salience network associated with stable global efficiency. Since local efficiency reflects the capacity for regional specialization in a network, it is plausible that dopaminergic treatment in PD-ICD patients specifically drives the functional reorganization of integrated brain networks, enhancing the aberrant coupling and looping of the reward system.

The present study has some limitations. Similar to other studies in the field $[9,10]$, the sample size can be considered relatively small. However, our groups were individually matched for age, sex, education and IQ in order to reduce variability. Although the fact that patients were studied on medication can be considered a limitation, we chose this study design because ICD is a complication associated with dopaminergic treatment. In addition, topological properties are not 
affected by medication [11] and dopaminergic medication did not differ between groups or show any association with the temporal properties of dynamic FNC. Finally, even if the duration of the resting state session and the movement threshold might be considered somewhat liberal for studies in healthy populations, we have used the same methodology than studies of dFNC in special populations with neurodegenerative diseases (Alzheimer disease, frontotemporal dementia and PD patients) $[15,16]$.

In conclusion, abnormal temporal patterns and stronger local topological properties in PD-ICD patients suggest a dynamic functional engagement of local connectivity involving the limbic circuit, leading to the inefficient modulation in emotional processing and reward-related decision-making. Moreover, these FNC alterations are not only coupled to impulsivity but also to a strong novelty seeking personality, suggesting that the above-mentioned functional abnormalities contribute to such traits. These findings suggests that a reorganization of the brain occurs in these patients such that regions involved in affection, reward processing and goaldirected behavior acquire a more significant role relative to other neurocognitive processes. We believe this analysis of the temporal dynamics of functional connectivity provide new insights into the pathophysiology of ICD in PD patients and that could be a useful imaging biomarker to identify PD patients at risk to develop ICD. 


\section{Financial disclosures}

Conflict of interest: Dr Rodriguez-Oroz received honoraria for lectures, travel and accommodation to attend scientific meetings from Abbvie, Zambon, Bial and Boston Scientific, and she received financial support for her research from national and local government funding agencies in Spain (Institute of Health Carlos III, Basque Country Local Government, and CIBERNED). None of the bodies influenced the content of the manuscript or the decision to publish in any way.

Funding: This work was supported by the Carlos III Institute of Health [PI11/02109] and by the ERA-NET Neuron program [PIM2010ERN-00733]. I.N-G. held a Mobility of Research Staff 2017 grant [MV17/00015] from the Carlos III Institute of Health under the framework of a Rio Hortega 2016 grant [CM16/00033]. J.K. has no disclosures to declare. P.M.P-A was supported by grants from the Spanish Ministry of Economy and Competitiveness [MINECO, RYC-201415440 and PGC2018-093408-B-I00], and Neuroscience Research Projects programme from the Fundación Tatiana Pérez de Guzmán el Bueno. BCBL acknowledges funding from the Basque Government through the BERC 2018-2021 program and by the Spanish State Research Agency through BCBL Severo Ochoa excellence accreditation [SEV-2015-0490]. A.P.S. is supported by the Canadian Institute of Health Research and Canada Research Chair program. M.C.R-O. received financial support for her research from national and local government institutions in Spain (Institute of Health Carlos III, Basque Country Government, Diputacion Foral Guipuzcoa and CIBERNED). 


\section{REFERENCES.}

[1] V. Voon, T.C. Napier, M.J. Frank, V. Sgambato-Faure, A.A. Grace, M. Rodriguez-Oroz, J. Obeso, E. Bezard, P.-O. Fernagut, Impulse control disorders and levodopa-induced dyskinesias in Parkinson's disease: an update, Lancet Neurol. 16 (2017) 238-250. doi:10.1016/S1474-4422(17)30004-2.

[2] F. Javoy-Agid, Y. Agid, Is the mesocortical dopaminergic system involved in Parkinson disease?, Neurology. 30 (1980) 1326-1330.

[3] R. Cilia, S.S. Cho, T. van Eimeren, G. Marotta, C. Siri, J.H. Ko, G. Pellecchia, G. Pezzoli, A. Antonini, A.P. Strafella, Pathological gambling in patients with Parkinson's disease is associated with fronto-striatal disconnection: A path modeling analysis, Mov. Disord. 26 (2011) 225-233. doi:10.1002/mds.23480.

[4] N. Carriere, R. Lopes, L. Defebvre, C. Delmaire, K. Dujardin, Impaired corticostriatal connectivity in impulse control disorders in Parkinson disease, Neurology. 84 (2015) 21162123. doi:10.1212/WNL.0000000000001619.

[5] M. Politis, C. Loane, K. Wu, S.S. O’Sullivan, Z. Woodhead, L. Kiferle, A.D. Lawrence, A.J. Lees, P. Piccini, Neural response to visual sexual cues in dopamine treatment-linked hypersexuality in Parkinson's disease, Brain. 136 (2013) 400-411. doi:10.1093/brain/aws326.

[6] R. Biundo, L. Weis, S. Facchini, P. Formento-Dojot, A. Vallelunga, M. Pilleri, D. Weintraub, A. Antonini, Patterns of cortical thickness associated with impulse control disorders in Parkinson's disease: NEUROANATOMICAL CORRELATES OF ICDS IN PD, Mov. Disord. 30 (2015) 688-695. doi:10.1002/mds.26154.

[7] H. Jiménez-Urbieta, B. Gago, P. de la Riva, M. Delgado-Alvarado, C. Marin, M.C. Rodriguez-Oroz, Dyskinesias and impulse control disorders in Parkinson's disease: From pathogenesis to potential therapeutic approaches, Neurosci. Biobehav. Rev. 56 (2015) 294314. doi:10.1016/j.neubiorev.2015.07.010.

[8] K. Petersen, N. Van Wouwe, A. Stark, Y.-C. Lin, H. Kang, P. Trujillo-Diaz, R. Kessler, D. Zald, M.J. Donahue, D.O. Claassen, Ventral striatal network connectivity reflects reward learning and behavior in patients with Parkinson's disease, Hum. Brain Mapp. (2017). doi:10.1002/hbm.23860.

[9] A. Tessitore, G. Santangelo, R. De Micco, A. Giordano, S. Raimo, M. Amboni, F. Esposito, P. Barone, G. Tedeschi, C. Vitale, Resting-state brain networks in patients with Parkinson's disease and impulse control disorders, Cortex. 94 (2017) 63-72. doi:10.1016/j.cortex.2017.06.008.

[10] A. Tessitore, R. De Micco, A. Giordano, F. di Nardo, G. Caiazzo, M. Siciliano, M. De Stefano, A. Russo, F. Esposito, G. Tedeschi, Intrinsic brain connectivity predicts impulse control disorders in patients with Parkinson's disease, Mov. Disord. Off. J. Mov. Disord. Soc. 32 (2017) 1710-1719. doi:10.1002/mds.27139.

[11] Z. Ye, A. Hammer, T.F. Münte, Pramipexole Modulates Interregional Connectivity Within the Sensorimotor Network, Brain Connect. 7 (2017) 258-263. doi:10.1089/brain.2017.0484.

[12] A. Verger, E. Klesse, M.B. Chawki, T. Witjas, J.-P. Azulay, A. Eusebio, E. Guedj, Brain PET substrate of impulse control disorders in Parkinson's disease: A metabolic connectivity study, Hum. Brain Mapp. (2018). doi:10.1002/hbm.24068. 
[13] V.D. Calhoun, R. Miller, G. Pearlson, T. Adal1, The chronnectome: time-varying connectivity networks as the next frontier in fMRI data discovery, Neuron. 84 (2014) 262274. doi:10.1016/j.neuron.2014.10.015.

[14] R.M. Hutchison, T. Womelsdorf, E.A. Allen, P.A. Bandettini, V.D. Calhoun, M. Corbetta, S. Della Penna, J.H. Duyn, G.H. Glover, J. Gonzalez-Castillo, D.A. Handwerker, S. Keilholz, V. Kiviniemi, D.A. Leopold, F. de Pasquale, O. Sporns, M. Walter, C. Chang, Dynamic functional connectivity: promise, issues, and interpretations, NeuroImage. 80 (2013) 360-378. doi:10.1016/j.neuroimage.2013.05.079.

[15] J. Kim, M. Criaud, S.S. Cho, M. Díez-Cirarda, A. Mihaescu, S. Coakeley, C. Ghadery, M. Valli, M.F. Jacobs, S. Houle, A.P. Strafella, Abnormal intrinsic brain functional network dynamics in Parkinson's disease, Brain J. Neurol. 140 (2017) 2955-2967. doi:10.1093/brain/awx233.

[16] M. Díez-Cirarda, A.P. Strafella, J. Kim, J. Peña, N. Ojeda, A. Cabrera-Zubizarreta, N. Ibarretxe-Bilbao, Dynamic functional connectivity in Parkinson's disease patients with mild cognitive impairment and normal cognition, NeuroImage Clin. 17 (2018) 847-855. doi:10.1016/j.nicl.2017.12.013.

[17] E. Fiorenzato, A.P. Strafella, J. Kim, R. Schifano, L. Weis, A. Antonini, R. Biundo, Dynamic functional connectivity changes associated with dementia in Parkinson's disease, Brain J. Neurol. (2019). doi:10.1093/brain/awz192.

[18] R. Girard, I. Obeso, S. Thobois, S.A. Park, T. Vidal, E. Favre, M. Ulla, E. Broussolle, P. Krack, F. Durif, J.-C. Dreher, Wait and you shall see: sexual delay discounting in hypersexual Parkinson's disease, Brain J. Neurol. 142 (2019) 146-162. doi:10.1093/brain/awy298.

[19] D. Weintraub, S. Hoops, J.A. Shea, K.E. Lyons, R. Pahwa, E.D. Driver-Dunckley, C.H. Adler, M.N. Potenza, J. Miyasaki, A.D. Siderowf, J.E. Duda, H.I. Hurtig, A. Colcher, S.S. Horn, M.B. Stern, V. Voon, Validation of the questionnaire for impulsive-compulsive disorders in Parkinson's disease, Mov. Disord. Off. J. Mov. Disord. Soc. 24 (2009) 14611467. doi:10.1002/mds.22571.

[20] D. Weintraub, E. Mamikonyan, K. Papay, J.A. Shea, S.X. Xie, A. Siderowf, Questionnaire for impulsive-compulsive disorders in Parkinson's Disease-Rating Scale, Mov. Disord. 27 (2012) 242-247. doi:10.1002/mds.24023.

[21] I. Litvan, J.G. Goldman, A.I. Tröster, B.A. Schmand, D. Weintraub, R.C. Petersen, B. Mollenhauer, C.H. Adler, K. Marder, C.H. Williams-Gray, D. Aarsland, J. Kulisevsky, M.C. Rodriguez-Oroz, D.J. Burn, R.A. Barker, M. Emre, Diagnostic criteria for mild cognitive impairment in Parkinson's disease: Movement Disorder Society Task Force guidelines, Mov. Disord. Off. J. Mov. Disord. Soc. 27 (2012) 349-356. doi:10.1002/mds. 24893.

[22] S. Whitfield-Gabrieli, A. Nieto-Castanon, Conn: a functional connectivity toolbox for correlated and anticorrelated brain networks, Brain Connect. 2 (2012) 125-141. doi:10.1089/brain.2012.0073.

[23] Roweis, ST, EM Algorithms for PCA and SPCA, (1998).

[24] E.A. Allen, E.B. Erhardt, E. Damaraju, W. Gruner, J.M. Segall, R.F. Silva, M. Havlicek, S. Rachakonda, J. Fries, R. Kalyanam, A.M. Michael, A. Caprihan, J.A. Turner, T. Eichele, S. Adelsheim, A.D. Bryan, J. Bustillo, V.P. Clark, S.W. Feldstein Ewing, F. Filbey, C.C. Ford, K. Hutchison, R.E. Jung, K.A. Kiehl, P. Kodituwakku, Y.M. Komesu, A.R. Mayer, G.D. Pearlson, J.P. Phillips, J.R. Sadek, M. Stevens, U. Teuscher, R.J. Thoma, V.D. Calhoun, A baseline for the multivariate comparison of resting-state networks, Front. Syst. Neurosci. 5 (2011) 2. doi:10.3389/fnsys.2011.00002. 
[25] B. Fischl, A. van der Kouwe, C. Destrieux, E. Halgren, F. Ségonne, D.H. Salat, E. Busa, L.J. Seidman, J. Goldstein, D. Kennedy, V. Caviness, N. Makris, B. Rosen, A.M. Dale, Automatically parcellating the human cerebral cortex, Cereb. Cortex N. Y. N 1991. 14 (2004) 11-22.

[26] W.R. Shirer, S. Ryali, E. Rykhlevskaia, V. Menon, M.D. Greicius, Decoding subject-driven cognitive states with whole-brain connectivity patterns, Cereb. Cortex N. Y. N 1991. 22 (2012) 158-165. doi:10.1093/cercor/bhr099.

[27] B. Rashid, E. Damaraju, G.D. Pearlson, V.D. Calhoun, Dynamic connectivity states estimated from resting fMRI Identify differences among Schizophrenia, bipolar disorder, and healthy control subjects, Front. Hum. Neurosci. 8 (2014) 897. doi:10.3389/fnhum.2014.00897.

[28] J.S. Nomi, S.G. Vij, D.R. Dajani, R. Steimke, E. Damaraju, S. Rachakonda, V.D. Calhoun, L.Q. Uddin, Chronnectomic patterns and neural flexibility underlie executive function, NeuroImage. 147 (2017) 861-871. doi:10.1016/j.neuroimage.2016.10.026.

[29] W.W. Seeley, V. Menon, A.F. Schatzberg, J. Keller, G.H. Glover, H. Kenna, A.L. Reiss, M.D. Greicius, Dissociable intrinsic connectivity networks for salience processing and executive control, J. Neurosci. Off. J. Soc. Neurosci. 27 (2007) 2349-2356. doi:10.1523/JNEUROSCI.5587-06.2007.

[30] R. Steimke, J.S. Nomi, V.D. Calhoun, C. Stelzel, L.M. Paschke, R. Gaschler, T. Goschke, H. Walter, L.Q. Uddin, Salience network dynamics underlying successful resistance of temptation, Soc. Cogn. Affect. Neurosci. 12 (2017) 1928-1939. doi:10.1093/scan/nsx123.

[31] S. Chiang, A. Cassese, M. Guindani, M. Vannucci, H.J. Yeh, Z. Haneef, J.M. Stern, Timedependence of graph theory metrics in functional connectivity analysis, NeuroImage. 125 (2016) 601-615. doi:10.1016/j.neuroimage.2015.10.070. 
Tables and figure legends

Table 1. Sociodemographic, clinical and behavioral characteristics of the sample

\begin{tabular}{|c|c|c|c|c|c|}
\hline & $\begin{array}{c}\text { PDICD } \\
\mathrm{n}=16\end{array}$ & $\begin{array}{c}\text { PDnoICD } \\
\mathbf{n}=20\end{array}$ & $\begin{array}{c}\mathbf{H C} \\
\mathbf{n}=17\end{array}$ & $p$ & $\begin{array}{c}\text { Post-hoc (Bonferroni or } \\
\text { U Mann Whitney) }\end{array}$ \\
\hline Age & $61.25(8.1)$ & $62.45(8.8)$ & $63.6(9.9)$ & $0.843^{\mathrm{a}}$ & \\
\hline Sex, male (\%) & $14(87.5 \%)$ & $16(84.2 \%)$ & $14(82 \%)$ & $0.834^{\mathrm{b}}$ & \\
\hline Education (years) & $16[9-18]$ & $12[9-13]$ & $15[8-20]$ & $0.470^{\mathrm{c}}$ & \\
\hline $\begin{array}{l}\text { Premorbid IQ (WAIS- } \\
\text { III Vocabulary) }\end{array}$ & $45.4(9.4)$ & $43.9(13.8)$ & $49.3(8.3)$ & $0.325^{\mathrm{a}}$ & \\
\hline $\begin{array}{l}\text { Disease duration } \\
\text { (years) }\end{array}$ & $7.47(4)$ & $6.65(4.6)$ & - & $0.583^{\mathrm{d}}$ & \\
\hline UPDRS-III & $20.31(9.6)$ & $25.90(7)$ & - & $0.122^{\mathrm{d}}$ & \\
\hline H\&Y stage & $2[1.5-2.5]$ & $2[1.5-3]$ & - & $0.492^{\mathrm{b}}$ & \\
\hline LEDD $_{\text {DA }}$ & $395[57.75-400]$ & $300[37.5-300]$ & - & $0.838^{\mathrm{e}}$ & \\
\hline LEDD $_{\text {TOTAL }}$ & 1210 [613-1449] & $725[487-1131]$ & - & $0.089^{\mathrm{e}}$ & \\
\hline PDQ-8 & $7.69(4.9)$ & $5.25(2.9)$ & $0.41(0.9)$ & $0.0001^{\mathrm{a}}$ & $\begin{array}{c}\text { PDICD }>\text { HC }(p=0.0001) \\
\text { PDnoICD }>\text { HC }(p=0.0001)\end{array}$ \\
\hline MoCA & $27(2.2)$ & $27.3(3)$ & $28.3(1.7)$ & $0.444^{\mathrm{a}}$ & \\
\hline HADS total & $8[5-18.2]$ & $4[1.2-5.7]$ & $5[4-7]$ & $0.015^{c}$ & $\begin{array}{c}\text { PDICD }>\text { PDnoICD } \\
(p=0.007) \\
\text { PDICD }>\text { HC }(p=0.048)\end{array}$ \\
\hline HADS-anxiety & $4.5(3.2)$ & $3.65(2.1)$ & $4.47(2.6)$ & $0.166^{\mathrm{a}}$ & \\
\hline HADS-depression & $3.5[1-5.7]$ & $1[0-2.7]$ & $1[0-2.5]$ & $0.003^{c}$ & $\begin{array}{c}\text { PDICD }>\text { PDnoICD } \\
(p=0.003) \\
\text { PDICD }>\text { HC }(p=0.004)\end{array}$ \\
\hline QUIP-RS score & $16(12.2-26.7)$ & - & - & - & \\
\hline $\begin{array}{l}\text { TCI-R Novelty Seeking } \\
\text { total }\end{array}$ & 99 [92.2-108.5] & 85 [73-92.7] & 91.7 [86.5-97] & $0.009^{c}$ & $\begin{array}{l}\text { PDICD }>\text { PDnoICD } \\
\quad(p=0.002)\end{array}$ \\
\hline $\begin{array}{l}\text { NS1 Exploratory } \\
\text { excitability }\end{array}$ & $29.2(7.1)$ & $25.1(7)$ & $27.7(4)$ & $0.148^{\mathrm{a}}$ & \\
\hline NS2 Impulsiveness & $25.8(6.6)$ & $18.1(6.5)$ & $23.5(5.3)$ & $0.002^{\mathrm{a}}$ & $\begin{array}{l}\text { PDICD }>\text { PDnoICD } \\
\quad(p=0.002)\end{array}$ \\
\hline NS3 Extravagance & $26.9(4.5)$ & $22.9(6.3)$ & $25.9(5.3)$ & $0.085^{\mathrm{a}}$ & \\
\hline NS4 Disorderliness & $18.3(6.4)$ & $14.5(5.1)$ & $16.6(3.8)$ & $0.033^{\mathrm{a}}$ & $\begin{array}{l}\text { PDICD }>\text { PDnoICD } \\
\quad(p=0.032)\end{array}$ \\
\hline $\begin{array}{l}\text { Barratt Impulsiveness } \\
\text { total }\end{array}$ & $40[28.2-61.2]$ & $29.5[25.2-34.5]$ & 33.5 [27.5-40.5] & $0.020^{a}$ & $\begin{array}{c}\text { PDICD }>\text { PDnoICD } \\
\quad(p=0.020)\end{array}$ \\
\hline Barratt Attention & $13.9(6.7)$ & $9.4(4.4)$ & $12.8(4.5)$ & $0.031^{a}$ & $\begin{array}{l}\text { PDICD }>\text { PDnoICD } \\
\quad(p=0.040)\end{array}$ \\
\hline $\begin{array}{l}\text { Barratt Motor } \\
\text { Impulsivity }\end{array}$ & $15.3(7.6)$ & $10.1(5)$ & $13.4(5)$ & $0.024^{a}$ & $\begin{array}{l}\text { PDICD }>\text { PDnoICD } \\
\quad(p=0.037)\end{array}$ \\
\hline Barratt Non-planning & $14.8(8.6)$ & $9.6(3.8)$ & $11.2(5.7)$ & $0.045^{a}$ & $\begin{array}{c}\text { PDICD }>\text { PDnoICD } \\
(p=0.042)\end{array}$ \\
\hline Starkstein score & $4.5[1-14.7]$ & $2.5[1-6.7]$ & $0[0-3]$ & $0.283^{\mathrm{c}}$ & \\
\hline
\end{tabular}

Values expressed in mean (SD) in parametric variables, in median and IQ range in non-parametric variables

${ }^{a}$ ANOVA

${ }^{\mathrm{b}}$ Chi-Square 
${ }^{\mathrm{c}}$ Kruskall-Wallis

${ }^{\mathrm{d}}$ Two-sample T-test

${ }^{\mathrm{e}} \mathrm{U}$ Mann Whitney

Abbreviations: IQ = Intelligence Quotient; WAIS-III = Wechsler Adult Inteligence Scale-III; UPDRS = Unified Parkinson's Disease Rating Scale; H\&Y=Hoehn and Yahr scale; LEDD $_{\text {TОтAL }}=$ Total daily levodopa equivalent dose. LEDD was calculated using the following formula LED $(\mathrm{mg})=(1 \mathrm{x}$ levodopa $)+[0.77 \mathrm{x}$ levodopa controlled release $(\mathrm{CR})]+(1.43 \mathrm{x}$ levodopa + entacapone $)+(1.11 \mathrm{x}$ levodopa $\mathrm{CR}+$ entacapone $)+(20 \mathrm{x}$ ropinirole $)+(20 \mathrm{x}$ ropinirole $\mathrm{ER})+(100 \mathrm{x}$ pramipexole $)+(30 \mathrm{x}$ rotigotine $)+(10 \mathrm{x}$ bromocriptine $)+(8 \mathrm{x}$ apomorphine $)+(100 \mathrm{x}$ pergolide $)+(67 \mathrm{x}$ cabergoline $)$ (Parkin et al., 2002$) ;$ LEDD $_{\mathrm{DA}}$ : daily levodopa equivalent dose of dopamine agonist according to the same formula; HADS=Hospital Anxiety and Depression Scale; ; QUIP-RS= Questionnaire for Impulsive-Compulsive Disorders in Parkinson's Disease-Rating Scale; TCI-R=Revised Temperament and Character Inventory; NS=Novelty Seeking Subscales. 
Figure 1. The 40 independent components (ICs) identified by a group ICA. (A) Spatial maps of the 40 ICs divided into seven networks. (B) Connectogram representing the connections between the ICs. Yellow lines represent positive functional connectivity, and blue lines represent negative functional connectivity (C) Group averaged static functional connectivity between IC pairs was computed using the entire resting state data. The value in the correlation matrix represents the Fisher's z-transformed Pearson correlation coefficient. Each of the 40 IC was rearranged by network group based on the seven functional networks. Index numbers of independent components are written on the left and bottom side of the matrix. Correlations between the ICs are represented in a scale from red (higher) to blue (lower).

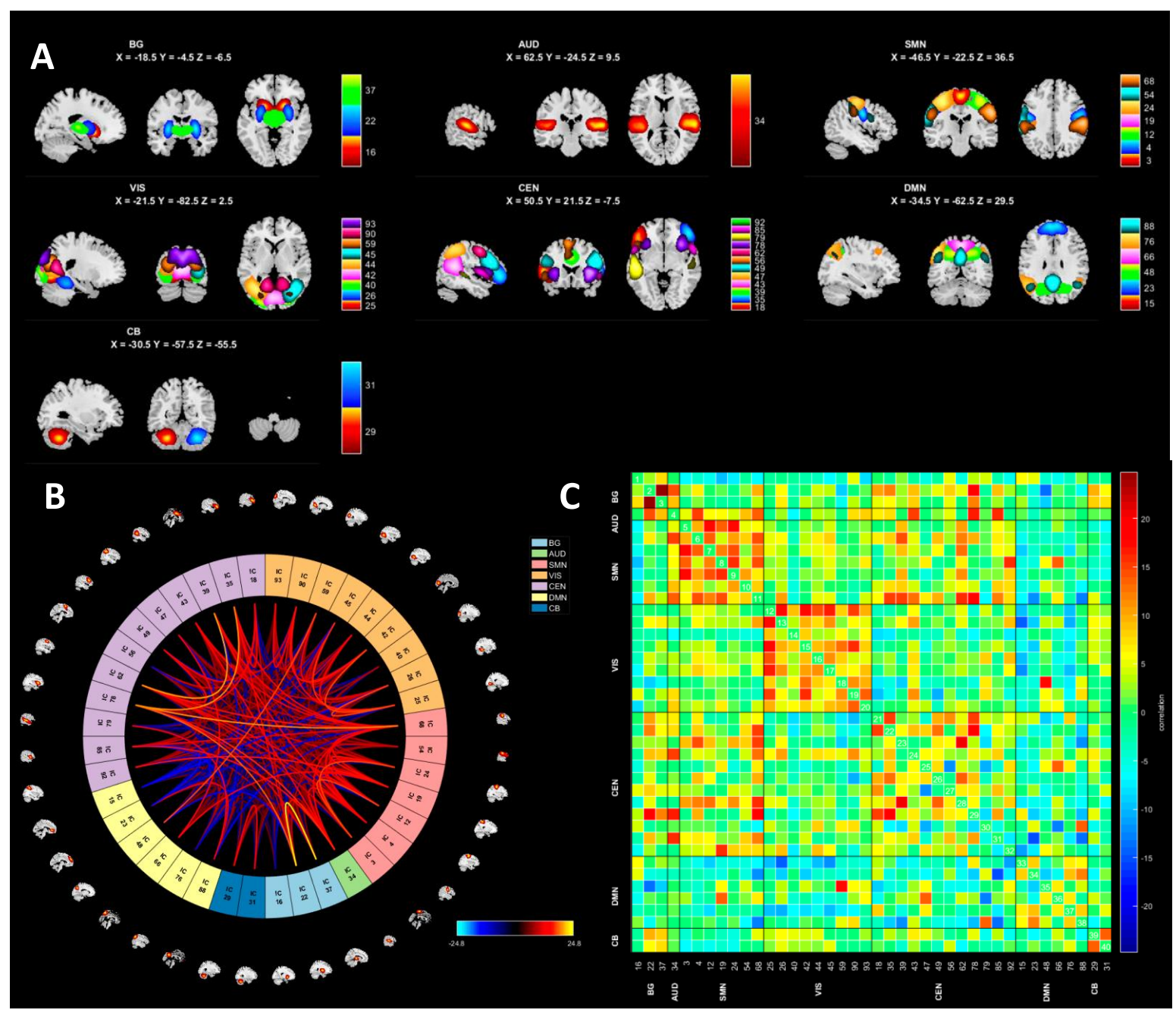


Figure 2. Dynamic FNC states resulting from the clustering analysis. A) Functional network connectivity matrices for each state, indicating the total occurrences and the percentages. Correlations between the ICs are represented in a scale from red (higher) to blue (lower). B) Connectogram representing the connections between the ICs (Yellow lines represent positive functional connectivity, and blue lines represent negative functional connectivity). C) Only that $5 \%$ of the functional connectivity matrix, representing the strongest connections (i.e. the largest absolute value correlation coefficients) is shown for each state. Red lines represent positive functional connectivity, and blue lines represent negative functional connectivity.
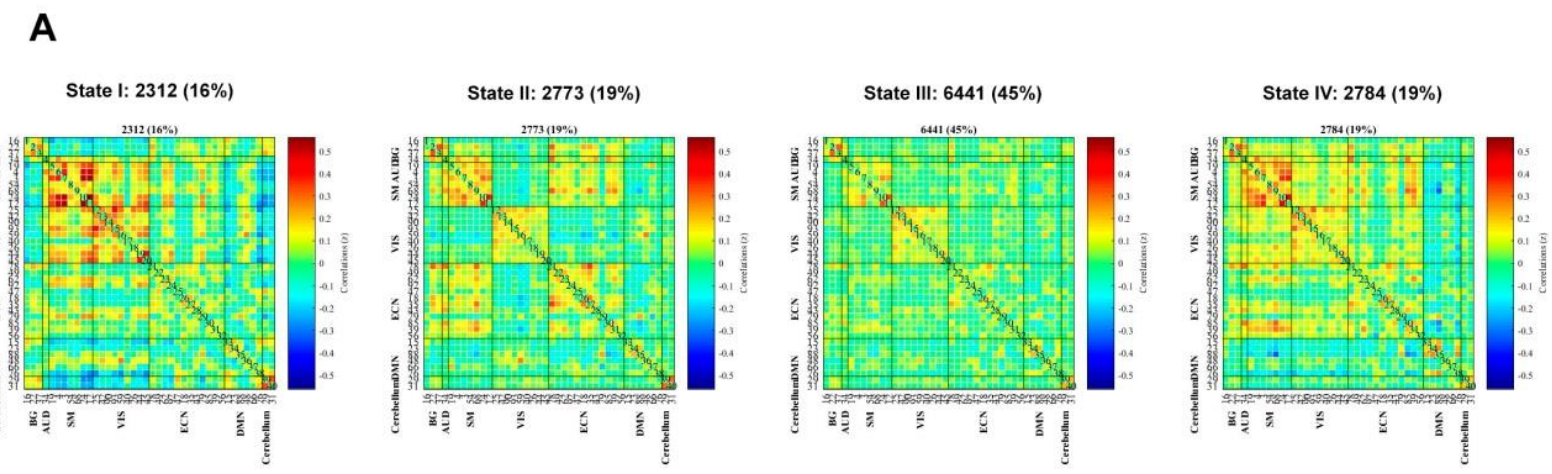

B

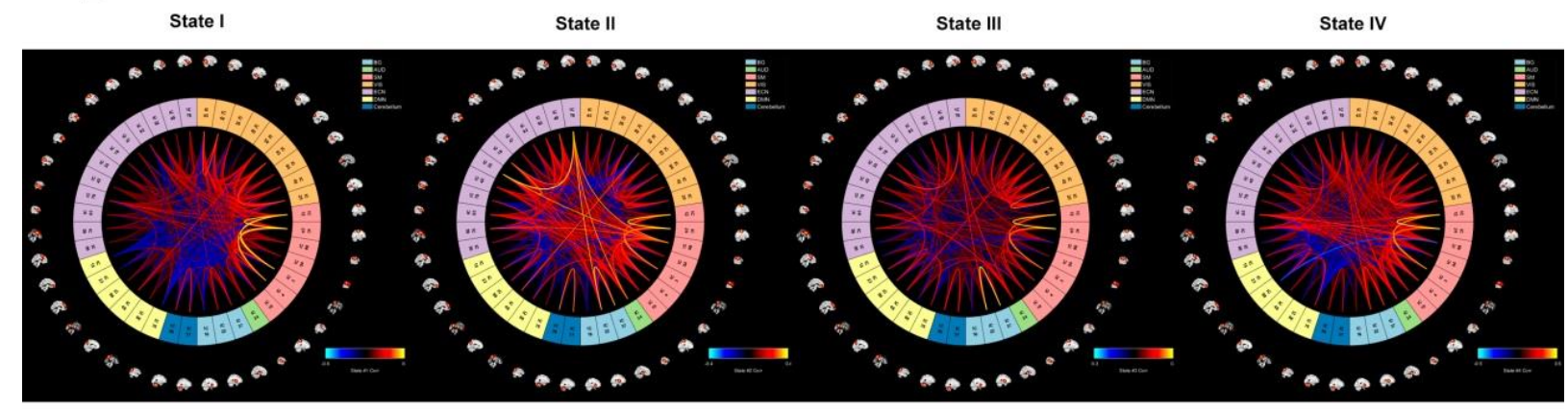

C
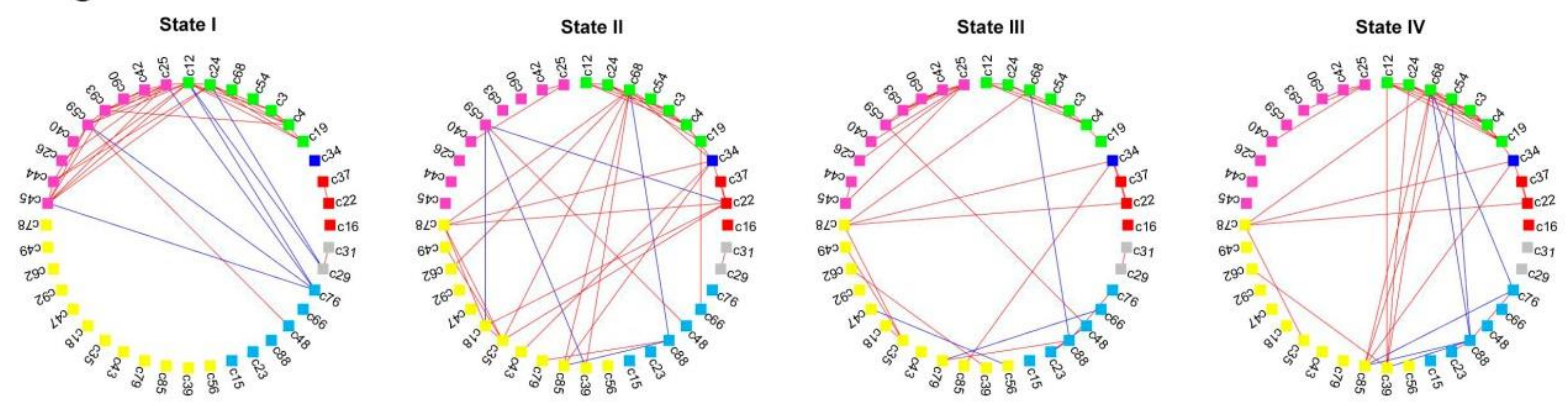
Figure 3: A) Differences in the temporal properties of the dynamic functional network connectivity states between the groups. (1) Mean dwell time (DT) between the groups and the significant differences between the groups in State III $(\&=$ PD-ICD $>H C ; \$=P D-I C D>P D-$ noICD) (2) Mean fractional window (FW) spent by the subjects in each state. B) Correlation of the clinical features of the PD-ICD group with the temporal properties detected. (1) The mean dwell time (DW) of State III was positively correlated with the NS sub-scale of the TCI-R scale (Novelty Seeking) and with the non-planning sub-scale of the BIS scale (Barratt Impulsiveness scale). (2) The fractional window (FW) of State III was positively correlated with the non-planning sub-scale and with the total BIS score.

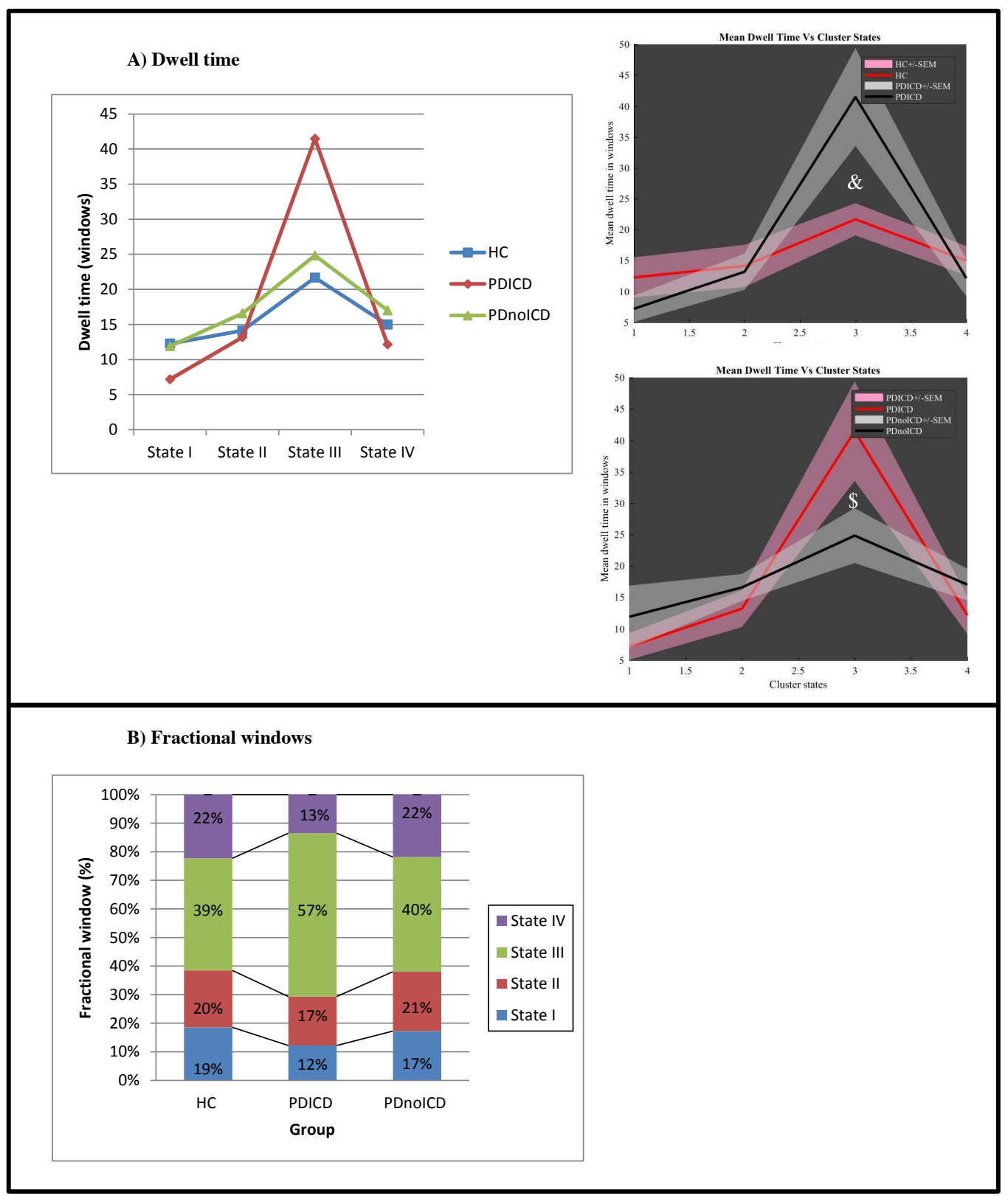

